index will, I think finally yield results. In tuberculin treatment we must bear in mind that we are dealing with an infectious disease, the cure for which depends on the establishment of complete immunity against the tubercle bacilli and its toxin. We must build up the patient so that his body cells will respond when stimulated. Next, we must see that our antibodies when present are applied. In small foci the small doses suggested by Wright are of value, but in advanced cases $I$ believe that the larger doses are more effective, and with increasing dosage and constant stimulation the largest amount of antibodies are formed.

Careful consideration of clinical symptom and close control of the patient, when using tuberculin, give the better results. I wish to see the patient on the day following as well as on the day on which the injection is made. To give the injections at set intervals is irrational. The time for injection depends on the size of the dose and the symptoms of the patient. The symptoms which guide me most often are unaccompanied by temperature rise. If the patient seems a little more nervous, if he aches, loses appetite, coughs and expectorates more than usual, I take it as a reaction. v. Pirquet's cutaneous test is very valuable, but requires considerable judgment. The symptomatology should aid you in deciding whether or not you are dealing with an active tuberculosis. If a closed case, you can inform your patient how to live.

Dr. Josferi McFarland, PhiladeIphia: Dr. Pottenger and I stand on opposite sides of this question. It is a matter of surprise to me that one so exact as Dr. Pottenger should be so extremely inaccurate in the matter of tuberculin. Personally, I am opposed to the use of tuberculin in the treatment of tuberculosis and I base that opposition on the fact that no animal can be imnunized against tuberculosis by any previous treatment with tuberculin, nor can any animal already tuberculous have its life considerably lengthened by tuberculin. Therefore why we should infer that anything experimentally impossible in the lower animals should prove successful in human beings I cannot understand. We know tuberculin is injurious, it has its place in making the diagnosis of tuberculosis in cows, it is said to be harmless when used for that purpose, and in assisting in diagnosis in man, but beyond that $I$ cannot see its usefulness. When Dr. Pottenger says that he has seen good results acerue from tuberculin that is entirely a matter of opinion, there is no measuring rod, there is no standard of comparison. Some time ago in discussing this matter with Dr. Flick I suggested that if the Phipps Institute could definitely establish some standard of comparison by which the different classes of cases could be investigated with reference to what they would do if they were treated dietically and climatologically without the use of medicaments of any kind, especially without the employment of tuberculin, it would be a most excellent work.

Dr. Theodone PotTer, Indianapolis: In reference to tuberculin, we connot insist too much, in connection with what is going on over the country, on the importance of clinical jucig. ment. Unless we do tlere is going to be a vast amount of reckless sort of diagnosis. It is plain that a considerable percentage of apparently perfectly healthy people react to tuberculin, and that there are others who may have a little focus that has been encapsulated and they react easily, and unless we insist to the average doctor that the mere application of tuberculin will not furnish a basis for his clinical diagnosis of tuberculosis, we shall have a lot of trouble over the country.

The surface tests vary in their rapidity. The eye test shows up most' quickly of all. 'That is, I believe, generally agreed to. It not infrequently begins to show in four to six hours and very frequently in eight to ten hours. There is in that fact sometimes an advantage; the time element in making a diagnosis is to the clinician sometimes important. For instance, some of you see cases from outside of the city. Patients come to the eity in the morning and must go home in the evening, or they come in the evening and think they must go home tomorrow morning, and in certain instances it seems proper to use tuberculin diagnosis. That may be the factor in determining the use of the eye test. As to the manner of action of tuberculin, its beneficial action: I happen to be one of those who have used tuberculin for many years, almost ever since it came out. I never believed and do not now believe that the only value is in its immunizing effect Dr. Baldwin referred to its stimulating the formation of the fibroid tissue, and I always have and now hold that is one of the distinctly valuable effects of tuberculin, but I cannot prove it. I have met with a limited number of interesting examples of most remarkable improvement following the use of one or two large doses of tuberculin which $I$ have never been able to explain. The artícle of Roteh of Boston, about a year ago in THE Journal on the diagnosis by tuberculin, instances a case in which two children were given by mistake very large doses of tuberculin, in each instance developing a temperature of 106. One of these clildren promptly died probabiy, he thought, as the result of the injection. The other child promptly proceeded to get better very rapidly after that large dose. Now I have happened to have several instances of that kind. For instance, a man came to me last October, now some 18 or 20 months ago; he was then 37 years of age. I had not seen him for a year or two, I saw at once that he was not well. He gave the typical history of tuberculosis, fever, night sweats, loss of weight from 160 to 130 or 140 , some 25 pounds loss. In spite of the most searching care I conld not localize the lesion. I gave him 2 mgrms. of tuberculin with what $I$ thought was a very moderate, but not very certain reaction. I then gave him an injection of 7 mgrms. of tested tuberculin, with a somewhat furious reaction, a temperature of 104 or more, and then there developed some slight focal symptoms, just recognizable, and that was all. I do not know where the real tuberculous focus was in that man. 1 recognized a little friction in one lung; he must have had tuberculosis somewhere else, probably in his bronchial glands. That man had been going for some three or four months down hill and without other treatment the man turned around and proceeded in a remarkable way to get well. He went along from the first of October until about the next April, when he showed some evidence of trouble and this performance was repeated. I gave him an injection of 4 mgrms. of tuberculin, just one, and he proceeded to get better and he is to-day apparently well. Now I have in the course of twelve or fifteen years happened to have had six or eight experiences of this sort in connection with the diagnostic use of tuberculin, all of course in early cases in which I could not be certain of the diagnosis from the ordinary physical method, and there occurred a pronounced reaction and forthwith there started a rather remarkable improvement. After fifteen years in the use of tuberculin to some moderate extent all the way along and more actively lately, I confess I do not know whether it is of much use or not.

\section{THE TREATMENT OF INOPERABLE SARCOMA BY ERYSIPELAS AND PRODIGIOSUS TOXINS *}

\section{LEO LOEB, M.D. PHILADELPHia}

The treatment of inoperable sarcoma by the toxins of certain bacteria is a treatment by vaccine. This vaccine differs, however, from certain other vaccines in not being a specific remedy, the toxins of the streptococci and of the Bacillus prodigiosus bearing no etiologic relation whatever to sarcoma, for the cure of which they are employed.

The foundation for this method of treatment of sarcoma is an empirical one. In a considerable number of cases in patients afflicted with cancer an attack of erysipelas led to a retrogression and in a certain number of cases to a total disappearance of the cancer, and especially of sarcoma. But -certain other acute infectious diseases may likewise cause a retrogression of cancer.

* Read in the Section on Pharmacology and Therapeutics of the American Medical Association, at the Sixtieth Annual Session. held at Atlantic City, June, 1909. 
Soon after the discovery of a streptococcus as the cause of erysipelas by Fehleisen ${ }^{1}$ this investigator inoculated patients who suffered from cancer with the cultures of streptococcus of erysipelas; he was able to obtain some beneficial results. (His first report appeared in the year 1882.) Other surgeons likewise reported cures. In all of these cases, however, living bacteria were used, and it is not to be wondered at that in some instances such inoculations were followed by the death of the patient.

At that time it was already known that toxins are mainly responsible for the pathogenic action of microorganisms. It was a step in advance when in 1891 Lassar, ${ }^{2}$ and soon afterward Spronck, ${ }^{3}$ in Utrecht, recommended the use of the toxins of streptococci and of erysipelas instead of the living germs. In $1891 \mathrm{~W}$. B. Coley of New York published some observations on the curative effect of the inoculation with the living streptococci of erysipelas in cases of sarcoma. In the following year he began a systematic study of the effect of the toxins of the streptococcus of erysipelas in the treatment of sarcoma. Since that time Dr. Coley has persistently continued in this line of therapeutical effort, and he has been able to collect very large series of cases treated by himself and by others with this method."

He improved the method of treatment by adding the toxins of the Bacillus prodigiosus to those of the Streptococcus erysipelatis. He was led to this change by the observations of Roger, who found that the toxins of the Bacillus prodigiosus increased, when injected into a rabbit, the virulence of living streptococci. In man, however, the cell contents of the Bacillus prodigiosus are toxic in themselves and the result of the injection of the combined toxins represents in all probability only the additive effect of both toxins. After various changes in the technic Dr. Coley found it best to use the non-filtered bouillon cultures of the streptococcus sterilized by heat, to which a certain amount of a culture of Bacillus prodigiosus, grown separately on agar and likewise sterilized by heat, had been added. The amount of endotoxin present in such preparations is greater than in filtered extracts. Later it was found that the Streptococcus myogenes isolated from cases of septicemia was as effective as the streptococcus isolated from cases of erysipelas. It is even likely that toxins of other bacteria might serve a similar purpose; and Beebe and Tracy found that the toxins of Bacillus coli had a similar effect on sarcoma of logs. And, indeed, as early as in the year 1895 Répin $^{2}$ reported on the use of cobra venom for the same purpose.

The toxins are injected in gradually increasing doses, at first in a part of the body removed from the tumor, and later, if possible, directly into the tumor itself. The usual effect of bacterial toxins-rise in temperature, malaise, signs of local inflammation-follow the injection. In favorable cases Dr. Coley finds that the tumor shrinks and becomes softer within a week after the beginning of treatment. A tumor which does not respond to the treatment within four weeks is not likely to be favorably influenced at a later period.

\footnotetext{
1. Fehleisen: Deutsch. med. Wchnschr., 1882, viii.

2. Isassar: Deutsch. med. W chnschr., 1891, No. 29.

3. Spronck : Ann. de l'Inst. Pasteur, 1892

4. Coley, William B.: Am. Jour. Med. Sc., March, 1906 ; The Treatment of Sarcoma with the Mixed Toxing of Erysipelas and Bacillus Prodigtosus, Bostan Med. and Surg. Joup., exvili, No. 6; Sarcoma of the Long Bones, Ann. Surg., March, 1907.

5. Roger; G. H. : Les maladies infeetleuses, Paris, 1902.

6. Beebe, S. P.. and Tracy, Martha : The Treatment of Experi. mental Tumors with Bacterial Toxins, THE Joursal A. M. A., Nov. 2, 1907 , No. 18, xlix, 1493.

7. Répin : Rev. de chir, 1805, xv.
}

According to Dr. Coley, the indications for the use of the toxins are the following:

1. All cases of inoperable sarcoma.

2. Certain cases of sarcoma of the long bones, still in the operable stage. In these the injection of the toxins may prevent the necessity of an amputation of the limb.

3. After operation for sarcoma, the injection being used as a routine treatment, in order to prevent recurrence of the tumor.

As to the actual results obtained with this treatment the following can be stated:

In tumors of dogs Spronck found in two cases no beneficial results; in five cases improvement followed the injection at a place distant from the tumor; the treatment, however, was not continued long enough to observa complete disappearance of the tumors. Spronek used spontaneous tumors. Beebe and Tracy observed a disappearance of the lymphosarcoma of dogs in a number of cases in which the toxins were injected into the tumor tissue itself. In one early case the tumor did not entirely disappear under the influence of the toxins and internal metastases were found at the post-mortem examination. It is to be emphasized, however, that these latter investigators did not use spontaneous but implanted dog tumors for their experiments, and it is certain that such inoculated tumors retrogress much more frequently spontaneously than primary tumors and are therefore probably more easily influenced by various therapeutic measures.

If we now consider the results obtained by this method of treatment in patients afflicted with sarcoma, Dr. Coley gives the following data:. In 430 cases treated the tumor disappeared under the influence of the toxins in approximately 11 per cent. of the cases; 6.5 per cent. of the cases have remained.without recurrence over three years after cessation of treatment. In 3 out of these 430 cases death followed, probably as a direct or indirect result of the treatment. According to Dr. Coley, in thirteen cases of sarcoma of long bones, observed partly by himself and partly by other surgeons, the use of the toxins has rendered amputation of the limb unnecessary; in other cases, however, the toxin treatment was without effect.

In a series of 22 cases in which the toxins were used after primary operation, 4 patients are now well after periods of three to eight years, and 9 after periods of one to three years; in 5 cases recurrence took place in spite of the toxin treatment; the remaining patients are still under treatment or the cases are very recent.

In order to supplement these statistics I have written to a number of prominent surgeons asking for a statement concerning their experience with Coley's fluid. Fourteen of these surgeons had had personal experience with this mode of treatment. The majority state, without giving the number of patients treated, that they have not seen any successful cases. From some surgeons I obtained the number of cases treated, and the result was as follows: Among 78 cases of sarcoma, in 4 cases a cure was obtained; therefore in not quite 5 per cent. of the cases treated a positive result was observed. On the other hand, in a number of cases in which no cure was obtained, the injection of the toxins seemed to have a marked weakening influence on the patient, and sometimes it produced a sloughing of the tumor.

It is therefore likely that the treatment of inoperable sarcoma with the toxins of streptococcus and Bacillus prodigiosus leads to a cure in approximately 4 to 9 per 
cent. of cases. And some results obtained so far suggest that this method of treatment may prove of value as a postoperative procedure in diminishing the number of recurrences, and that in a certain number of cases it might limit the necessity for amputation of the limb in cases of sarcoma of the long bones. As to its mode of action, nothing definite can be stated, but it is likeiy that the toxins themselves, as well as the local and general reactions they produce, frequently affect the life of the sarcoma cells unfavorably.

\section{ABSTRACT OF DISCUSSION}

Dr. B. A. Thomas, Philadelphia: Dr. Loeb's paper has recalled to my mind a very interesting case which was brought to my attention a year since. The patient had been suffering for several weeks from an inoperable malignant tumor of the mouth and was being treated at the time by one of Philadelphia's most eminent internists, whose word I think would substantiate the diagnosis of a definite neoplasm. After several weeks of treatment the patient was told that nothing further could be done and was advised to arrange his estate. A few days later he was taken ill with a severe infection of grippe. He was confined to bed for several days. A few weeks after he recovered he called to see the physician again and was informed that, beyond a doubt, there had been a considerable decrease in the size of the tumor and there was evidence of healing. In the course of a few weeks the growth had entirely disappeared and the patient died subsequently of other intercurrent condition. It seems to me advisable to report this case, in the belief that toxins of bacteria or infections other than those caused by Streptococcus or B. prodigiosus may at times be instrumental in the cure or treatment of inoperable sarcoma.

Dr. W. Duffield RoBinson, Philadelphia: I employed Coley's serum in a case of extraperitoneal sarcoma and had tried everything else I could think of and all had failed absolutely in doing anything, but the Coley serum had the most remarkable control of pain. The young man required $11 / 2$ grains of morphin every hour or two; on the second day after beginning the treatment the morphin was reduced to $1 / 4$, at the end of two weeks $1 / 12$ grain three times a day. He was made much more comfortable and improved in many ways, but eventually died. Coley changed the serum at different times according to his ideas of the condition present. The entire treatment was under Coley's supervision. The other case was sarcoma of the neck and jaw. This patient was taken to the hospital and treated under Dr. Coley's supervision with out result, except for controlling of pain. It failed entirely, although the serum was crowded to the maximum dose. Operation was done and the man has a recurrence now.

De. 0. P. Johnstone, Pittsburg, Pa.: I have used Coley's fluid in two cases of sarcoma; one, a typical large spindlecelled sarcoma of the palate, had been operated on three times the previous year, with recurrences. After the third operation I recommended a trial of Coley's fluid, and it was given every day for three months. It is now a year since the last operation and there has been no recurrence. In the second case, a spindle-celled sarcoma of the skin, operated on a year ago, could not be completely removed, sections from the edge of the portion removed still showing tumor tissue. Coley's fluid was given daily for eight months, and twice a week for the following four months. The wound healed nicely, and the skin around the wound where the sarcoma tissue was left appears normal. The girl has increased in weight and appeers perfectly well a year after the operation.

Plague Infected Wood Rat.-The discovery of a wood rat (Neotoma Fuscipes anectens, Elliot), from Alameda county, California, on Oct. 17, 1909, infected with bubonic plague, adds a new link to the chain of plague, as it is believed this is the first plague-infected wood rat ever discovered. The wood rat may act as an intermediary in the transmission of disease to other mammals. The infection was found by Passed Assistant Surgeon G. W. McCoy of the laboratory in San Francisco (Public Health Reports, Jan. 7, 1910).

\section{ANTIVENINS* \\ IIIDEYO NOGUCHI, M.D. \\ NEW YORK}

In order to understand the proper administration of antivenins we must first consider several fundamental facts concerning the main properties of snake venoms and their antitoxins.

\section{PROPERTIES OF VENOMS}

1. Constituents.-There are three principal groups of death-dealing constituents in snake venoms, namely, the neurotoxins, hemorrhagins and fibrin ferments. In the venoms of colubrine snakes the neurotoxins are the most important constituents, while the hemorrhagins constitute the chief toxins of all the viperine venoms. The fibrin ferments are present in the colubrine as well as the viperine venoms and vary somewhat with the species. These three groups of toxins may in most venoms be present in varying quantities, but some venoms contain almost exclusively the neurotoxins, or neurotoxins and fibrin ferments with but little hemorrhagin. Thus the venoms of the Australian snakes-Pseudechis and Notechis-contain all three constituents in fairly even quantity, those of the marine snakes only the neurotoxins, those of the Indian and African colubrine snakes chiefly the neurotoxins with a negligible amount of hemorrhagins. The venoms of crotaline snakes, including the rattlesnakes and pit-vipers of America and Asia, contain chiefly hemorrhagins, with secondary amounts of the neurotoxins and fibrin-ferments. The real vipers owe their poisonousness to hemorrhagins, and sometimes to powerful fibrin ferments in their venoms. The most deadly of all the true vipers are the Indian Daboia and Echis. The Elaps of America has a venom rich in neurotoxins.

2. The Causes of Death.-Death from snake-poisoning is due to various causes, according to the varieties of venoms introduced. The death from the cobras, Elaps, Bungarus and marine snake-bite is due to the paralysis of respiratory center. The fatal issue from the poisoning by the daboia, Echis, and Australian snakes is due either to a rapid intravascular thrombosis or to secondary poisoning or infection resulting in marasmus. In cases of rattlesnake or any other crotaline snake-bite death is caused by occasional hemorrhages in vital organs or by a setting in of secondary poisoning resulting in cachexia or septicemia. In excessive absorption of these venoms deatl may result from the effects of neurotoxins also. 'The most distinctive of venom toxication in the crotaline bite is the extensive local disturbances produced by the hemorrhagins; this local effect.is highly important in considering all the viperine and crotaline poisoning.

3. Fatal Dosage.-The minimal fatal doses of different venoms can be accurately determined by animal experimentation. But this is influenced by the mode of introduction of snake venom into the animal body. Thus in case of neurotoxic venom there is but little difference in the final result whether it is introduced directly into the circulation or under the skin. On the other hand, the minimal lethal dose of fibrin ferment containing venoms is very much smaller when injected into the circulation than when introduced subcutaneously, the greater part of the toxic ferment being absorbed in the neighborhood of the injection site. This is also true

* Read in the Section on Pharmacology and 'rinerapeutics of the American Medical Association, at the Sixtieth Annual Session, held at Atlantic City, June, 1909. 